\title{
Genetic encoding of targeted MRI contrast agents for in vivo tumor imaging
}

Simone Schuerle ${ }^{1}$, Maiko Furubayashi ${ }^{2,3}$, Ava P. Soleimany ${ }^{4,5,6}$, Tinotenda Gwisai ${ }^{1}$, Wei Huang $^{4}$, Christopher Voigt ${ }^{2}$, Sangeeta N. Bhatia ${ }^{4,6,7,8,9,10,11,12}$

\section{Affiliations}

1. Institute for Translational Medicine, Department of Health Sciences and Technology, ETH Zurich, $\mathrm{CH}-8092$ Zurich, Switzerland.

2. Synthetic Biology Center, Department of Biological Engineering, Massachusetts Institute of Technology, Cambridge, MA 02139, USA.

3. Bioproduction Research Institute, National Institute of Advanced Industrial Science and Technology (AIST), Sapporo, Japan.

4. Koch Institute for Integrative Cancer Research, Massachusetts Institute of Technology, Cambridge, MA 02139, USA.

5. Harvard Graduate Program in Biophysics, Harvard University, Boston, MA 02115

6. Harvard-MIT Division of Health Sciences and Technology, Institute for Medical Engineering and Science, Massachusetts Institute of Technology, Cambridge, MA 02139, USA.

7. Research Laboratory of Electronics, Massachusetts Institute of Technology, Cambridge, MA 02139, USA.

8. Electrical Engineering and Computer Science, Massachusetts Institute of Technology, Cambridge, MA 02139, USA.

9. Marble Center for Cancer Nanomedicine, Massachusetts Institute of Technology, Cambridge, MA 02139, USA.

10. Department of Medicine, Brigham and Women's Hospital and Harvard Medical School, Boston, MA 02115, USA.

11. Broad Institute of Massachusetts Institute of Technology and Harvard, Cambridge, MA 02139, USA.

12. Howard Hughes Medical Institute, Cambridge, MA 02139, USA.

${ }^{*}$ Corresponding Author: Sangeeta N. Bhatia

Address: Massachusetts Institute of Technology, 77 Massachusetts Avenue, Building 76453, Cambridge, MA 02139, USA.

Phone: +16173240610

Email: sbhatia@mit.edu 


\section{Abstract}

Tumor-selective contrast agents have the potential to aid in the diagnosis and treatment of cancer using noninvasive imaging modalities such as magnetic resonance imaging (MRI). Such contrast agents can consist of magnetic nanoparticles incorporating functionalities that respond to cues specific to tumor environments. Genetically engineering magnetotactic bacteria to display peptides has been investigated as a means to produce contrast agents that combine the robust image contrast effects of magnetosomes with transgenic targeting peptides displayed on their surface. This work reports the first use of magnetic nanoparticles that display genetically-encoded $\mathrm{pH}$ low insertion peptide (pHLIP), a long peptide intended to enhance MRI contrast by targeting the extracellular acidity associated with the tumors. To demonstrate the modularity of this versatile platform to incorporate diverse targeting ligands by genetic engineering, we also incorporated the cyclic av integrin-binding peptide iRGD into separate magnetosomes. Specifically, we investigate their potential for enhanced binding and tumor imaging both in vitro and in vivo. Our experiments indicate that these tailored magnetosomes retain their magnetic properties, making them well-suited as T2 contrast agents, while exhibiting increased binding compared to wild-type magnetosomes.

Keywords: magnetosomes, magnetotactic bacteria, tumor targeting peptides, MRI, synthetic biology 


\section{Introduction}

Robust imaging of tumors for diagnosis and treatment monitoring has the potential to improve healthcare outcomes and save lives. Wide recognition of this principle, combined with established screening practices based on noninvasive imaging modalities, has spurred investigation into contrast agents that aid in distinguishing tumors from surrounding tissue. ${ }^{1,2}$ Among possible imaging modalities, magnetic resonance imaging (MRI) is especially appealing because magnetic fields are relatively innocuous compared to ionizing radiation, and targets throughout the body can be readily resolved. ${ }^{3}$ Contrast agents designed to identify tumors do so by exploiting their unique physical and biochemical characteristics. An effective tumor-selective imaging agent must therefore combine properties that make it robustly detectable via the imaging modality with features that lead to preferential accumulation or enhanced contrast effects in tumors. Because targeting strategies often rely upon biomarkers specific to particular types of tumors, tumor-selective contrast agents based on generalized characteristics of tumors are especially desirable.

MRI contrast agents typically function by detectably altering the longitudinal (T1) or transverse (T2) relaxation times of nearby hydrogen nuclei. ${ }^{3}$ Synthetic magnetic nanomaterials, especially ferrite nanoparticles, have been deployed as MRI contrast agents due to their magnetic properties and biocompatibility. For instance, size-dependent surface effects in ultrasmall ferrite nanoparticles have spurred their investigation as T1 contrast agents, offering a potential alternative to the paramagnetic gadolinium ion complexes currently employed. ${ }^{4}$ The use of somewhat larger iron oxide nanoparticles as T2 contrast agents is well established, with examples of such particles clinically approved as T2 contrast agents. ${ }^{3}$ Synthetic biology has also offered schemes for in vivo molecular imaging with MRI, including imaging tumors via selective expression of MRI contrast reporter genes or by modulating endogenous ferritin expression. ${ }^{5-8}$ In another study, expression of genetically encoded reporters based on gas vesicles for hyperpolarized xenon magnetic resonance imaging was shown in living cells with potential use for in vivo imaging via systemic injection of tumor-targeted encoded viral vectors. ${ }^{9}$ Nanomaterial synthesis and synthetic biology intersect with the intriguing concept of harnessing magnetotactic bacteria (MTB), a group of prokaryotes known for their ability to biomineralize pristine intracellular nanocrystals of magnetite $\left(\mathrm{Fe}_{3} \mathrm{O}_{4}\right)$ with sizes ranging from $35-120 \mathrm{~nm}$, as a biogenic source of high quality T2 contrast agents. ${ }^{10-12}$ These particles occur in chains and are surrounded by a phospholipid bilayer membrane, forming a structure called a magnetosome. Recent research demonstrated the display of targeting peptides on the surfaces of these magnetosomes, such as RGD and recently the GFR/HER2 targeting peptide P75. ${ }^{13-16}$ As targeting moieties, peptides offer several advantages, including their small size, high affinity, ease of modification, and low immunogenicity. ${ }^{17-20}$ Despite their promise, some peptides are challenging to attach to the surface of synthetic iron oxide nanoparticles while maintaining their structural confirmation, making transgenic expression on the surface of magnetosomes the most direct means to produce contrast agents in such cases. ${ }^{21,22}$

This work provides the first report of magnetic nanoparticles displaying genetically-encoded $\mathrm{pH}$ low insertion peptide (pHLIP) ${ }^{23}$, a long peptide that is ill suited for chemical conjugation. Targeting mechanisms based on $\mathrm{pH}$ responsiveness hold particular promise as broadly tumor-selective MRI contrast agents, since they exploit the extracellular acidity associated with the tumor microenvironment 
for contrast enhancement. ${ }^{24}$ In separate genetically engineered magnetosomes, we incorporated the av integrin-binding cyclic peptide iRGD, which is known to specifically target tumors by binding integrinexpressing cells in a neuropilin-1-dependent manner. Upon binding to av integrins on the endothelium of tumor vessels, it is proteolytically cleaved within the tumor microenvironment, increasing affinity for neuropilin-1 and facilitating tissue penetration of co-administered or conjugated drugs or imaging agents. ${ }^{25,26}$ In addition to verifying the applicability of this technique for functionalization of magnetosomes with widely different peptide ligands, this approach provided a means to compare the performance of pHLIP against a peptide with known tumor targeting and penetration capabilities. We found increased binding affinity of both pHLIP- and iRGD-functionalized magnetosomes to cancer cells in low $\mathrm{pH}$ culture conditions, or to cancer cells expressing av integrins, respectively, relative to nonfunctionalized magnetosomes. Further, this selectivity was also observed in vivo during experiments in which functionalized magnetosomes accumulated at tumor sites and produced measurable local decreases in T2 relaxation times. Because genetic engineering allows different peptides to simultaneously be displayed on individual magnetosomes, our findings suggest that pHLIP and iRGD could serve as useful components of a tailored peptide combination on magnetosomes acting as generalized tumor-selective T2 contrast agents.

\section{Results and Discussion}

\section{Purified magnetosomes are suitable T2-relaxation contrast agents}

To date, a range of magnetotactic bacteria strains have been discovered that all appear to synthesize magnetosomes. In this work, we employed Magnetospirillum magneticum AMB-1 cells, for which the complete genome sequence is available. ${ }^{27}$ Before undertaking the genetic modification of these bacteria to tag their magnetosomes with custom peptides in order to improve their targeting as tumor contrast agents, the structure and magnetic properties of AMB-1 and their extracted and purified magnetosomes were investigated. Using cryo-transmission electron microscopy (cryo-TEM), AMB-1 were imaged prior to magnetosome extraction to verify the formation of magnetosome chains which were then analyzed in more detail after harvesting and purification (see Materials and Methods). We observed $200-500 \mathrm{~nm}$ long chains of individual iron oxide crystals, encompassed by a $5-6 \mathrm{~nm}$ proteinrich phospholipid bilayer that made up the magnetosome membrane (Fig. 1a). The micrographs of the magnetosomes purified in this study showed individual grain sizes on the order of $40 \mathrm{~nm}$, a size consistent with the range encountered in wild-type MTB and suggestive of their function as carriers of high-stability natural remanence. At physiological temperatures and long timescales, the critical size above which individual grains of cubic magnetite are no longer superparamagnetic is $25-30 \mathrm{~nm},{ }^{28}$ and pseudo single domain and multidomain behavior is expected at lengths above approximately $80 \mathrm{~nm}{ }^{29}$ The size of the isolated particles falls between these values, suggesting they should exist in a stable single domain (SSD) state. This characterization was corroborated by observations of hysteresis at room temperature when vibrating sample magnetometry (VSM) was performed on samples prepared in gels to prevent their physical rotation (Fig. 1b). Because individual iron oxide crystals within a magnetosome chain are only separated by a few $\mathrm{nm}$, magnetostatic interactions have been observed to affect the orientation of the moment of each crystal, resulting in alignment along the axis of the 
chain. ${ }^{30}$ The same magnetic properties were retained for the purified magnetosomes, indicating that they maintained their structural integrity (Fig. 1b).

The magnetic properties of magnetosomes have prompted previous investigations into their suitability as contrast agents, both for magnetic particle imaging (MPI) and MRI. ${ }^{11}$ In a previous study, magnetosome contrast enhancement was determined to be slightly elevated compared to the former iron oxide-based "gold-standard", Resovist, with a hydrodynamic diameter between 45 and $60 \mathrm{~nm}$ and an iron oxide nanoparticle core between only 5 and $6 \mathrm{~nm} .{ }^{11,31}$ We measured an increased reduction of T2-weighted signal relaxation time of $17.3 \pm 2.6 \mathrm{~ms}$ for purified magnetosomes with an iron oxide core diameter of approximately $40 \mathrm{~nm}$ at an iron concentration of $0.2 \mathrm{mM}$. This effect on T2 relaxation time reduction exceeded that of commercial, well dispersed magnetite particles with a similar core diameter of $25 \mathrm{~nm}$, for which a value of $31.9 \pm 2.6$ ms was measured at the same iron concentration (Fig. 1c,d). This result is consistent with the capacity of purified magnetosomes to mediate a T2 relaxation time reduction and their suitability as a core material for functional MRI contrast agents.

\section{Engineered peptide-displaying magnetosomes retain wild-type magnetic properties}

After characterizing the properties of the native AMB-1 magnetosomes, we sought to genetically engineer AMB-1 bacteria to produce peptide-functionalized magnetosomes. The technique to display peptides on the surface of magnetosomes has been well established during the past decade. ${ }^{32,33}$ Several studies have suggested that a magnetosome-specific protein called mamC is the most abundant protein on the magnetosome surface ${ }^{34}$, and thus it has been used previously for display. As a preliminary validation, we sought to display a his-tag on the magnetosome. To do this, we fused the his-tag sequence to the C-terminal of the mamC gene immediately before the stop codon, and placed this tag under the regulation of the IPTG-inducible ptac promoter. The resulting plasmid was then conjugated into Magnetospirillum magneticum AMB-1. The proteins from the purified magnetosomes were collected and analyzed by SDS-PAGE and Western blotting (Fig 2a). These results indicate that our experimental protocols indeed fuse peptides to the magnetosomes.

We proceeded to design DNA sequences to display pHLIP and iRGD on magnetosomes to add relevant functionality and selectivity in context of tumor targeting contrast agents, both for acidic tumor tissues and integrin-expressing cancer cells, respectively. ${ }^{25,35-37}$ pHLIP and iRGD peptide-encoding DNA sequences were generated by backtranslating using a GC rich codon to optimize expression in M. magneticum. These DNA sequences were fused to the $\mathrm{C}$-terminus of the mamC gene immediately before the stop codon and after a linker sequence (Fig. 2b). Except for the peptide sequence, the construct is identical to the one validated for the his-tagged magnetosomes. The pHLIP- and iRGDencoding plasmids were conjugated into M. magneticum, and magnetosomes from the cells were collected, purified, and thoroughly washed. To verify that genetic modification of their surface proteins did not alter properties relevant to their function as T2 contrast agents, we acquired hysteresis curves for these genetically engineered pHLIP- and IRGD-magnetosomes, which yielded comparable results to the wild-type magnetosomes (Fig. 2c). The saturation magnetization, as determined by these measurements and quantification of iron content via inductively-coupled plasma mass spectrometry (ICP-MS), suggested values of $111 \mathrm{emu} / \mathrm{g}_{\mathrm{Fe}}$ for wild-type magnetosomes, $91.2 \mathrm{emu} / \mathrm{g}_{\mathrm{Fe}}$ for $\mathrm{iRGD}$ - 
expressing magnetosomes, and $96.3 \mathrm{emu} / \mathrm{g}_{\mathrm{Fe}}$ for pHLIP-expressing magnetosomes. These values indicate that the high saturation magnetization values of wild-type MTB magnetosomes are retained upon expression of IRGD or pHLIP and are consistent with the majority of iron in the particles occurring as biomineralized magnetite $\left(115 \mathrm{emu} / \mathrm{g}_{\mathrm{Fe}}\right.$ in bulk). ${ }^{38}$

\section{Peptide-fused magnetosomes serve as functional binding agents}

Next, we tested whether our selected peptides, pHLIP and iRGD, retained their functionality when displayed on the surface of purified magnetosomes. The unique characteristic of pHLIP is its ability to associate with lipid bilayers as an unstructured monomer at neutral $\mathrm{pH}$, while inserting across a bilayer or membrane as an alpha-helix in acidic conditions. As a result, magnetosomes displaying pHLIP are expected to show a pH-dependent fusion to cell membranes compared to unmodified magnetosomes. Thus, we cultured cells of the human breast cancer cell line MDA-MB-231, both at a standard $\mathrm{pH}$ of 7.5 and at a low, slightly acidic $\mathrm{pH}$ of 6.5 , which is representative of average tumor acidity. ${ }^{39}$ We fluorescently labeled magnetosomes with and without expression of pHLIP using a lipophilic membrane dye (Dil) inserted into the membrane of the magnetosome. We incubated both wild-type and pHLIP-functionalized magnetosomes with MDA-MB-231 cells at low $\mathrm{pH}$ and standard $\mathrm{pH}$ and analyzed the binding efficiency by flow cytometry. Our data revealed a significant increase in bound magnetosomes to cells for $\mathrm{pHLIP}$-functionalized magnetosomes at low $\mathrm{pH}$ compared to neutral $\mathrm{pH}$, as well as compared to pure magnetosomes at low $\mathrm{pH}$, demonstrating $\mathrm{pH}$-activated binding to cancer cells in vitro (Fig. 3a). As expected, there was no observable difference in the binding efficiency of pure magnetosomes with cancer cells between these two different $\mathrm{pH}$ levels.

Analogously, we assessed the functionality of iRGD displayed on magnetosomes by testing the specificity of binding to av $\beta 3$ expressing cells, such as the MDA-MB-231 cells used in the previous experiments. ${ }^{40,41}$ A 1.53-fold increase in binding efficiency was measured by flow cytometry for iRGDfunctionalized magnetosomes compared to wild-type magnetosomes (Fig. 3b). Analysis of fixed samples by confocal imaging also revealed the localization of the magnetosomes at the cell surface. Further, trypsin treatment, which has been shown to cleave cell-surface integrins non-specifically, ${ }^{42}$ resulted in a $42 \%$ decrease in the fluorescent intensity of iRGD-functionalized magnetosome binding, further supporting our hypothesis of integrin-selective targeting of iRGD-functionalized magnetosomes (Fig. 3b).

\section{Magnetosomes fused with tumor-targeting peptides serve as potential in vivo cancer imaging agents}

Finally, we tested peptide-functionalized and non-decorated magnetosomes in vivo by intravenously injecting suspensions of the respective purified and near infrared (NIR) fluorescentlylabeled magnetosomes into mice bearing flank xenografts of the human ductal carcinoma (mammary gland origin) cell line MDA-MB-435S. These cells strongly express the integrin $a v \beta 3,{ }^{43,44}$ and thus, we expected to observe an increase in tumoral accumulation of the iRGD-fused magnetosomes relative to non-decorated magnetosomes. To assess biodistribution, organs were harvested 6 and $24 \mathrm{~h}$ post injection and analyzed with a NIR scanner (Fig. 4a,b). Both pHLIP and iRGD peptide-functionalized 
magnetosomes showed a trend towards increased accumulation at tumor sites compared to pure magnetosomes; specifically, approximately 1.5 -fold and 2-fold increases in tumoral accumulation for pHLIP and iRGD, respectively, were measured after $6 \mathrm{~h}$ (Fig. 4c). This data is consistent with values found in comparable previous studies, including a large literature survey on tumor accumulation of nanoparticles that broadly suggest a 1.5 - to 2 -fold increase in tumor delivery for targeted versus nonspecific nanoparticles. ${ }^{17,45}$ A decrease in signal after $24 \mathrm{~h}$ suggested rapid clearance of the magnetosomes.

Based on these results, we decided to analyze the tumors within the first $6 \mathrm{~h}$ post injection with T2-weighted image analysis in a 7T whole mouse MRI scanner. A darkening of the tumor regions was observed for all magnetosome types, as shown in scans in Figure 5a, while the strongest enhancement was observed for iRGD-fused magnetosomes, in line with the results of the biodistribution analysis and similar to results obtained in vitro for the av $\beta 3$ expressing MDA-MB-231 cell line. We then quantified the impact on T2-relaxation time reduction, i.e. the extent to which iRGD-functionalized magnetosomes darken the targeted tumor region (Fig. 5b). Compared to wild-type magnetosomes, the decrease in relaxation time increased by 2.02 -fold, although not with statistical significance, with three animals in each group bearing two flank tumors.

To demonstrate the impact of low pH targeting in vivo, we characterized the performance of the pHLIP-functionalized magnetosomes in a flank tumor model of the LS174T colorectal cancer cell line, which only faintly expresses the av integrin. ${ }^{46}$ To further increase the potential contrast enhancement, we concentrated our magnetosome formation from $0.5 \mathrm{mg} / \mathrm{ml}$ to $1.5 \mathrm{mg} / \mathrm{ml}$ compared to the previous trial and injected $0.3 \mathrm{mg}$ per mouse. An increased accumulation at tumor sites was again measured for pHLIP-functionalized magnetosomes over non-functionalized magnetosomes (Fig. 5c, inset). The T2relaxation was reduced for pHLIP magnetosomes by $7.72 \%(6.40 \mathrm{~ms})$, however, not significantly across the small number of animals ( $n=3$ for the control and $n=4$ for the experimental group, Fig. $5 c$ ). An example scan of two flank tumors is shown in Figure $\mathbf{5 d}$ with an overlaid T2 relaxation time map, which was quantified across 6 slices (Fig. 5e). Follow up studies with larger cohorts of animals could be suggested for higher powered statistics; however, these results already indicate the potential of peptide functionalized magnetosomes as T2-weighted contrast agents for in vivo tumor imaging.

\section{Conclusion}

We have demonstrated the design, synthesis, and application of T2-weighted tumor imaging probes produced through genetic manipulation of MTB to display the tumor-targeting peptides pHLIP or iRGD on their magnetosome membranes. This genetic modification was found to leave the magnetic properties of these structures substantially unaltered. The intended functionality of both peptides was verified experimentally in vitro. Animal studies employing small numbers of mice also showed indications of tumor selectivity and enhanced T2-weighted contrast in vivo. The up to 2-fold enhancement in accumulation for targeted magnetosomes is consistent with the range of delivery enhancement previously suggested in literature for tumor targeting nanoparticles.

This approach combines the biomineralized cores of magnetosomes, along with their uniquely advantageous magnetic properties, with genetically engineered surface functionalizations that impart 
targeting and other useful capabilities. The versatility and flexibility to specifically tailor and rapidly synthesize new peptide-functionalized imaging probes with such pristine magnetic properties has great potential in clinical applications as targeted diagnostic agents, specifically when incorporating targeting peptides, that are complex to synthesize synthetically. The simultaneous incorporation of multiple peptides to obtain multiplexed functionality could open new avenues in multimodal diagnostics.

\section{Materials and Methods}

\section{Bacterial media composition}

Modified Magnetosome Growth (MMG) media was prepared by a slight modification of Magnetospirillum Growth Media (MSGM). ${ }^{47}$ First, MMG was prepared by adding the following to $1 \mathrm{~L}$ distilled water: $0.68 \mathrm{~g} \mathrm{KH}_{2} \mathrm{PO}_{4}$ (Fischer), $0.34 \mathrm{~g} \mathrm{NaNO}_{3}$ (Fischer), $0.37 \mathrm{~g}$ tartaric acid (Alfa-Aesar), 0.37 $\mathrm{g}$ succinic acid (Sigma-Aldrich), $0.05 \mathrm{~g}$ sodium acetate (Sigma-Aldrich), $5 \mathrm{~mL}$ ATCC Trace Mineral Solution. The $\mathrm{pH}$ was then adjusted to 6.8 by $10 \mathrm{~N} \mathrm{NaOH}$, and the solution was autoclaved at $121^{\circ} \mathrm{C}$ for $40 \mathrm{~min}$. The MMG could be kept on the shelf for $\sim 2$ months. Just before each bacterial culture, ATCC Vitamin Supplement (ATCC MD-VS, 100 to 1000x dilution), $10 \mathrm{mM}$ ferric quinate (100x dilution, final concentration of $100 \mu \mathrm{M}$ ), ascorbic acid (see "Bacterial strains and culture conditions" for the concentration), and appropriate antibiotics or inducers were freshly added to the MMG.

Agar media with final concentration of $0.8 \%$ agar was prepared by adding $8 \mathrm{~g}$ of Bacto Agar (Difco) to $1 \mathrm{~L} \mathrm{MMG-base} \mathrm{and} \mathrm{autoclaving} \mathrm{at} 121^{\circ} \mathrm{C}$ for $40 \mathrm{~min}$. After the media was cooled below $60^{\circ} \mathrm{C}$, ATCC Vitamin solution (1000x), $10 \mathrm{mM}$ ferric quinate (100x), and appropriate antibiotics were added and poured into a plastic dish. (Note that ascorbic acid is not included). The plates were stored at $4^{\circ} \mathrm{C}$, and warmed to $30^{\circ} \mathrm{C}$ just before plating.

Stock solutions were prepared as follows: $10 \mathrm{mM}$ ferric quinate (100x) was prepared by adding $0.27 \mathrm{~g}$ of $\mathrm{FeCl}_{3} 6 \mathrm{H}_{2} \mathrm{O}$ and $0.19 \mathrm{~g}$ of $\mathrm{D}$-(-)-quinic acid, sterile filtered, and used within 2 weeks. $1000 \mathrm{x}$ ascorbic acid solution ( $1 \mathrm{~g} / \mathrm{L}$ for seed culture or $20 \mathrm{~g} / \mathrm{L}$ for passage culture) was prepared and sterile filtered just before each culture. 100x 2,6-diaminopimelic acid (DAP) was prepared by adding $0.285 \mathrm{~g}$ of DAP to $100 \mathrm{~mL}$ distilled water while heating, followed by sterile filtration. Antibiotics used (in their final concentration) were $5 \mu \mathrm{g} / \mathrm{mL}$ Kanamycin and $5 \mu \mathrm{g} / \mathrm{mL}$ Gentamycin for liquid culture, or $10 \mu \mathrm{g} / \mathrm{mL}$ Kanamycin and $10 \mu \mathrm{g} / \mathrm{mL}$ Gentamycin for agar culture.

\section{Bacterial strains and culture condition}

E. coli NEB 10-beta (NEB) was used for cloning, and WM3064 (also known as BW29427) (thrB1004 pro thi rpsL hsdS lacZ $\Delta$ M15 RP4-1360 $\Delta$ (araBAD)567 $\Delta$ dapA1341::[erm pir], derived from W. Metcalf $)^{47}$ was used for plasmid conjugation. WM3064 harbors RP4 conjugation machinery in the genome, and lacks dapA gene and therefore requires DAP to grow.

M. magneticum AMB-1 was purchased from ATCC and cultures were started by streaking glycerol stocks on MMG agar or inoculating glycerol stocks directly into a liquid culture. After streaking the cells, the plates were inserted in campy pouch (BD) and incubated at $30^{\circ} \mathrm{C}$ for $5-7$ days to obtain single colonies. Tiny colonies are visible after 4-5 days, and growing them for additional 2-3 days (total 
7 days) resulted in fully-grown colonies. The plates were pulled out from the bag for further experiments. The plates were kept in a bag flushed with nitrogen to keep a microaerobic environment at room temperature and used within 1 week.

For the seed culture in liquid media, MMG media was prepared just before inoculation by adding the following to MMG-base (described in section "Bacterial media composition"): ATCC Vitamin solution (1000x), $0.1 \mathrm{~g} / \mathrm{L}$ ascorbic acid (1000x), $10 \mathrm{mM}$ ferric quinate (100x) and appropriate antibiotics or inducers. Seed culture was performed in $5 \mathrm{~mL}$ of MMG media using $10 \mathrm{~mL}$ gas vials. Single colonies were inoculated into the media, and the rubber-cap was tightened. The headspace was replaced with $\mathrm{N} 2$ by vacuuming the headspace and adding N2 gas, then repeated three times. $250 \mu \mathrm{L}$ of air (approximately $50 \mu \mathrm{L}$ of $\mathrm{O} 2$, which is $1 \%$ of the headspace) was injected into the headspace using a needle and a syringe. The vial was placed into a water bath at $30^{\circ} \mathrm{C}$ and shook at $60 \mathrm{rpm}$. This seed culture was incubated for 3 days.

Second-passage cultures were performed in either $5 \mathrm{~mL}$ or $100 \mathrm{~mL}$ culture. MMG media was prepared with slight modification, by adding 1000x $2 \mathrm{~g} / \mathrm{L}$ ascorbic acid (final concentration $20 \mathrm{mg} / \mathrm{L}$ ). For $100 \mathrm{~mL}$ culture, $100 \mathrm{~mL}$ MMG media using a $170 \mathrm{~mL}$ glass jar, sealed by rubber cap (SigmaAldrich). The cells were inoculated by 1:50 dilution (by adding $2 \mathrm{~mL}$ seed culture), the headspace was replaced with $\mathrm{N} 2,700 \mu \mathrm{L} \mathrm{O} 2$ was added, and cultures were incubated at $30^{\circ} \mathrm{C}$.

For large-scale culture (third-passage culture), 2.4 L MMG media was prepared in a $2.8 \mathrm{~L}$ flask. Fifty $\mathrm{mL}$ of second-passage culture was added, the flask was sealed with a rubber stopper, and the headspace was replaced with $1 \% \mathrm{O}_{2}$ and $99 \% \mathrm{~N}_{2}$ using a needle through the rubber stopper. The culture was cultured at $30^{\circ} \mathrm{C}, 60 \mathrm{rpm}$.

\section{Magnetosome purification}

Magnetosomes were purified from M. magneticum based on the method reported by Borg et al. ${ }^{34}$ with modifications. Four batches of $M$. magneticum culture were collected in $1 \mathrm{~L}$ bottles with centrifuge at $5000 \mathrm{~g}$ for $20 \mathrm{~min}$. The pellet from $1 \mathrm{~L}$ culture was resuspended with $60 \mathrm{~mL}$ of $20 \mathrm{mM}$ HEPES (pH 7.4) with $1 \mathrm{mM}$ EDTA (HEPES-E buffer), and $30 \mathrm{~mL}$ of culture was dispensed in two 50 $\mathrm{mL}$ tubes (or $60 \mathrm{~mL}$ culture was collected into $250 \mathrm{~mL}$ bottles), and centrifuged at $4700 \mathrm{~g}$ for $20 \mathrm{~min}$. The cell pellet was kept at $-80^{\circ} \mathrm{C}$ if not immediately used.

The cell pellet was resuspended in HEPES-E supplemented with $0.1 \mathrm{mM} \mathrm{PMSF}$ by vortexing. The resuspension was passed through a French Press at $20 \mathrm{kpsi}$. The lysate was transferred to 250 $\mathrm{mL}$ bottles, and was sonicated on ice for $1 \mathrm{~min}$ at $10 \%$ amplitude with 1-sec ON/OFF interval. Lysates were transferred to $50-\mathrm{mL}$ falcon tubes and placed next to a strong magnet overnight at $4^{\circ} \mathrm{C}$. The supernatant was aspirated, and the magnetic fraction was resuspended into 10-mL HEPES-E. The solution was sonicated on ice for $30 \mathrm{sec}$ at $10 \%$ amplitude with 1-sec ON/OFF interval. This was placed next to a strong magnet for 4 hours at $4^{\circ} \mathrm{C}$, and this washing step was repeated 3-10 times. After washing, the magnetic fraction was resuspended a final time in distilled water or PBS and kept at $4^{\circ} \mathrm{C}$ until use. 


\section{Plasmid construction}

Plasmids and genetic construct used in this study are described in Supplementary Table 1. pMGA vector was constructed by merging the PMGT vector ${ }^{48}$ and $\mathrm{p} 15 \mathrm{~A}$ plasmid. The mamC gene was PCR-amplified from the $M$. magneticum genome and inserted under the IPTG-inducible Ptac/Lacl promoter. The peptide sequence for the his-tag, iRGD and pHLIP were (in single-letter amino acid abbreviations) $\quad \mathrm{HHHHHHHHH}, \quad$ CRGDKGPDC 26 and AAEQNPIYWARYADWLFTTPLLLLDLALLVDADEGT ${ }^{35}$, respectively. The DNA sequence for these peptide tags were generated by backtranslating using the GC-rich Streptomyces coelicolor codon usage table. The pHLIP-tag were fused right after the C-terminal of mamC CDS, while the his-tag and iRGDtag were fused to mamC after an AGGS peptide linker. The DNA sequence of the fused mamC-peptide CDS are shown in Supplementary Table 2.

\section{Cell culture}

MDA-MB-231 and MDA-MB-435S cells were cultured in high glucose Dulbecco's Modified Eagle's Medium (DMEM, Gibco) supplemented with $10 \%$ foetal bovine serum (FBS) in a humidified atmosphere with $5 \% \mathrm{CO}_{2}$ at $37^{\circ} \mathrm{C}$. MDA-MB-435S cell cultures were supplemented with $1 \%$ penicillinstreptomycin (CellGro). LS174T were cultured in Eagle's Minimal Essential Medium (ATCC) supplemented with 10\% FBS (Gibco) and 1\% penicillin-streptomycin (CellGro). All cells were passaged at $80 \%$ confluency.

\section{Measurement of magnetic properties of MTB and purified magnetosomes}

A Microsense vibrating sample magnetometer (EZ VSM) measured moment versus field curves at room temperature for $100 \mu \mathrm{L}$ samples of MTB and magnetosomes prepared in modified NMR tubes. Samples were affixed with silicone vacuum grease to a vibrating sample rod shortened to ensure centering in the gap of the electromagnet between the sensing coils. Where indicated, to prevent physical rotation of MTB or magnetosomes, samples were prepared in 2 to $4 \%$ agarose gel by adding agarose powder to the samples, briefly microwaving them (30 to 60s) while held upright in a thin layer of water in a glass petri dish, and placing them on ice to gel. Diamagnetic signal was subtracted by linear fits of the data at high field magnitudes, and M/Ms curves were obtained by normalizing to the saturation moment indicated by the intercepts of these linear fits.

\section{In vitro evaluation of magnetosome targeting}

MDA-MB-231 cells were cultured to $80 \%$ confluency on coverslips in 6 -well plates and stained with $200 \mu \mathrm{L}$ complete DMEM containing $1 \mu \mathrm{L} \mathrm{DiO}$ at a concentration of $1 \mathrm{mM}$ (Sigma) to visualise the cell membrane. After $45 \mathrm{~min}$ incubation in a humidified atmosphere with $5 \% \mathrm{CO} 2$ at $37^{\circ} \mathrm{C}$, the cells were washed twice with 1X PBS. Functionalized magnetosomes were stained with Dil (Sigma), incubated at room temperature with gentle agitation for $30 \mathrm{~min}$ and were washed twice with PBS. For iRGD-fused magnetosomes, cells were treated with $400 \mu \mathrm{L}$ of magnetosomes in $1 \mathrm{~mL}$ complete DMEM on ice. For pHLIP-fused magnetosomes, cells were treated with $50 \mu \mathrm{L}$ of magnetosomes in $1 \mathrm{~mL}$ complete DMEM on ice at $\mathrm{pH} 6.5$ or 7.4 . The $\mathrm{pH} 6.5$ medium was prepared by adding $20 \mathrm{mM}$ HEPES 
and $20 \mathrm{mM}$ MES to complete DMEM. Cells treated with unfunctionalized magnetosomes were used as negative controls. After one hour incubation, the magnetosome solution was removed and cells were washed twice with PBS. The cells were then fixed with $4 \%$ paraformaldehyde (PFA) on ice for 5 min and then washed twice with PBS. The coverslips were mounted on glass slides and were viewed under a spinning disk confocal microscope.

\section{Quantification of magnetosome targeting by Flow cytometry}

For quantitative analysis of magnetosome binding, MDA-MB 231 cells were cultured to approximately $80 \%$ confluency. Cells were detached and transferred to tubes before treatment with stained functionalized magnetosomes. For iRGD-fused magnetosomes, 125,000 cells were treated with $25 \mu \mathrm{L}$ of magnetosomes in $500 \mu \mathrm{L}$ complete DMEM on ice. For pHLIP-fused magnetosomes, 500,000 cells were treated with $25 \mu \mathrm{L}$ of magnetosomes in $500 \mu \mathrm{L}$ complete DMEM on ice at pH 6.5 or 7.4. Cells treated with unfunctionalized magnetosomes were used as negative controls. After $1 \mathrm{~h}$ incubation, the magnetosome solution was removed and cells were washed twice with PBS. The cells were then fixed with $4 \%$ PFA on ice for $5 \mathrm{~min}$, washed twice with PBS and resuspended $2 \%$ bovine serum albumin (BSA) in PBS. Cells were analyzed using a $561 \mathrm{~nm}$ excitation laser and 586/15 filter on a BD LSRFortessa. Untreated cells were used to set the gate on live cells (FSC/SSC). The fluorescence emission of 10,000 cells was recorded and targeting data are reported as mean fluorescence intensity (MFI). For all samples and controls Student's t-tests were performed.

\section{ICP-MS}

Samples of $50 \mu \mathrm{l}$ were prepared in glass vials and evaporated overnight in a ventilated oven or on a hot plate. $200 \mu \mathrm{l}$ of concentrated $\mathrm{HCl}(37 \% \mathrm{w} / \mathrm{w})$ was added to dissolve the iron and diluted in a solution of $2 \% \mathrm{HNO} 3$ in purified water. Analysis was carried out using ICP-TOFMS instrument (icpTOF, TOFWERK AG, Switzerland) or ICP-MS (Agilent 7900 ICP-MS). A reference solution was used at the beginning, middle, and end of sample measurements as a quality control. For calibration, five reference solutions containing different concentrations of $\mathrm{Fe}$, and $\mathrm{Rh}$, Co or In as internal standards were prepared.

\section{In vivo cancer model studies}

Female nude mice (4-6 weeks, Taconic) were inoculated bilaterally with $3 \times 10^{6}$ MDA-MB-435S or LS174T cells per flanks. Tumor growth was monitored and 10-14 days after inoculation, biodistribution and MRI studies were scheduled. Purified magnetosomes were labeled with a near infrared (NIR) lipophilic membrane dye (DiR'; DilC18(7); 1,1'-Dioctadecyl-3,3,3',3'Tetramethylindotricarbocyanine lodide, ThermoFisher) at a volume ratio of 1:2000, washed three times, and suspended in PBS. Iron concentrations were assessed by ICP-MS, and solutions were adjusted to yield $0.5 \mathrm{mg} / \mathrm{ml} \mathrm{Fe}$ or $1.5 \mathrm{mg} / \mathrm{ml} \mathrm{Fe}$ (two trials) respectively. Suspensions were administered intravenously via tail vein injections at a maximum volume of $200 \mu \mathrm{l}$ and an iron mass of $0.1 \mathrm{mg}$ or 0.3 $\mathrm{mg}$. 


\section{Magnetic resonance imaging}

For MRI phantoms, serial dilutions of $0.4 \mathrm{mM}$ Fe stock suspensions of magnetosomes and commercially available IONPs (SHP-25, Ocean Nanotech) were prepared in PBS. $200 \mu \mathrm{l}$ of each sample was added into PCR tubes containing pre-weighted agar yielding final suspensions of $1 \mathrm{wt} \%$. Samples were thoroughly mixed and sonicated, heated in a microwave, and cooled on ice to allow gelling. Imaging phantoms were prepared by inserting PCR tubes into $50 \mathrm{ml}$ tubes, filling them with with $1 \mathrm{wt} \%$ agar, followed by heating in a microwave and gelling on ice. Phantom imaging experiments were then performed on a 9.4T small animal scanner (94/30 USR, Bruker, Ettlingen, Germany), equipped with a $87 \mathrm{~mm}$ quadrature volume resonator. Multi echo multi slice sequence (MEMS) was used to evaluate T2 relaxation time, with the following parameters: repetition time TR $=2000 \mathrm{~ms}$, number of echoes $=28$, echo time TE $=9 \mathrm{~ms}$ to $252 \mathrm{~ms}$ with a $9 \mathrm{~ms}$ increment, data matrix $=320 \times 120$, Field of View $(F O V)=80 \times 30 \mathrm{~mm}, 5$ slices, slice thickness $=1.5 \mathrm{~mm}, 4$ averages in $16 \mathrm{~min}$.

MRI experiments were performed on a 7T MRI whole mouse MRI system (Varian 7T/210/ASR, Varian/Agilent), equipped with a $38 \mathrm{~mm}$ mouse body coil. Multi echo multi slice sequence (MEMS) was used to evaluate T2 relaxation time, with the following parameters: repetition time TR $=1500 \mathrm{~ms}$, number of echoes $=28$, echo time TE $=9 \mathrm{~ms}$ to $252 \mathrm{~ms}$ with a $9 \mathrm{~ms}$ increment, data matrix $=128 \times 128$, Field of View $(F O V)=50 \times 50 \mathrm{~mm}$. T2 weighted images were acquired with fast spin-echo multi-slice sequence (FSEMS) with the following parameters: $T R=2000 \mathrm{~ms}, T E=24 \mathrm{~ms}$, $E T L=4$, kzero $=2$, $256 \times 256$ matrix, FOV $=50 \times 50 \mathrm{~mm}^{2}$, interleaved number of slices=20 with no gap and slice thickness $=0.5 \mathrm{~mm}$, number of averages $=2$. Images were converted to DICOM format for viewing and analyzing purposes.

During imaging, mice were anesthetized by inhalation of $2.5 \%$ isoflurane and maintained on $2 \%$ isoflurane during data collection. Hot air was delivered throughout the imaging session to provide heat. Scans were collected with respiratory gating (PC-SAM version 6.26 by SA Instruments Inc., Stony Brook, New York) to avoid confounding noise due to chest movement.

\section{Statistics and Data Analysis}

All statistical analyses were performed in GraphPad (Prism 8.0). Statistical significance and individual tests are described in figure legends.

\section{Acknowledgements}

We thank Dr. M.-A. Augath and Dr. A. Schröter from the Rudin Laboratory at ETHZ for assistance with MRI scans of phantoms, Dr. H. Fleming (MIT) and Dr. M. Christiansen (ETHZ) for critical reading and editing of the manuscript, Dr. L. Gonzalez and Dr. P. R. Leduc (CMU) for the pMGT plasmid, and the Koch Biopolymers \& Proteomics Core for assistance. We thank the Koch Institute Swanson Biotechnology Center for technical support, specifically S. Malstrom in the Koch Institute Animal Imaging and Preclinical Testing core. S.S. gratefully acknowledges the support by the Branco Weiss fellowship. The work from M.F. and C.A.V. was supported by the Institute for Collaborative Biotechnologies through contract W911NF-09-0001 and W911NF-19-2-0026 with the U.S. Army Research Office. A.P.S. thanks the NIH Molecular Biophysics Training Grant and the National Science 
Foundation Graduate Research Fellowship Program for support. S.N.B. is a Howard Hughes Medical Institute Investigator. This study was supported in part by a Koch Institute Support Grant P30-CA14051 from the National Cancer Institute (Swanson Biotechnology Center), a Core Center Grant P30ES002109 from the National Institute of Environmental Health Sciences, the Ludwig Fund for Cancer Research and the Koch Institute Marble Center for Cancer Nanomedicine.

\section{References}

1. Fass, L. Imaging and cancer: a review. Mol. Oncol. 2, 115-152 (2008).

2. Seaman, M. E., Contino, G., Bardeesy, N. \& Kelly, K. A. Molecular imaging agents: impact on diagnosis and therapeutics in oncology. Expert Rev. Mol. Med. 12, e20 (2010).

3. Gallagher, F. A. An introduction to functional and molecular imaging with MRI. Clin. Radiol. 65, $557-566$ (2010).

4. Kim, B. H. et al. Large-scale synthesis of uniform and extremely small-sized iron oxide nanoparticles for high-resolution T1 magnetic resonance imaging contrast agents. J. Am. Chem. Soc. 133, 12624-12631 (2011).

5. Louie, A. Y. et al. In vivo visualization of gene expression using magnetic resonance imaging. Nat. Biotechnol. 18, 321-325 (2000).

6. Gilad, A. A. \& Shapiro, M. G. Molecular Imaging in Synthetic Biology, and Synthetic Biology in Molecular Imaging. Mol. Imaging Biol. 19, 373-378 (2017).

7. Mukherjee, A., Davis, H. C., Ramesh, P., Lu, G. J. \& Shapiro, M. G. Biomolecular MRI reporters: Evolution of new mechanisms. Prog. Nucl. Magn. Reson. Spectrosc. 102-103, 32-42 (2017).

8. Minn, I. et al. Tumor-specific expression and detection of a CEST reporter gene. Magn. Reson. Med. 74, 544-549 (2015).

9. Shapiro, M. G. et al. Genetically encoded reporters for hyperpolarized xenon magnetic resonance imaging. Nat. Chem. 6, 629-634 (2014).

10. Alphandéry, E. Applications of magnetosomes synthesized by magnetotactic bacteria in medicine. Front Bioeng Biotechnol 2, 5 (2014).

11. Heinke, D. et al. MPS and MRI efficacy of magnetosomes from wild-type and mutant bacterial 
strains. International Journal on Magnetic Particle Imaging 3, (2017).

12. Lang, C. \& Schüler, D. Biogenic nanoparticles: production, characterization, and application of bacterial magnetosomes. J. Phys. Condens. Matter (2006).

13. Xiang, Z. et al. Tumor detection using magnetosome nanoparticles functionalized with a newly screened EGFR/HER2 targeting peptide. Biomaterials 115, 53-64 (2017).

14. Yan, L., Da, H., Zhang, S., López, V. M. \& Wang, W. Bacterial magnetosome and its potential application. Microbiol. Res. 203, 19-28 (2017).

15. Sangnier, A. P. et al. Targeted thermal therapy with genetically engineered magnetite magnetosomes@ RGD: Photothermia is far more efficient than magnetic hyperthermia. J. Control. Release 279, 271-281 (2018).

16. Boucher, M. et al. Genetically tailored magnetosomes used as MRI probe for molecular imaging of brain tumor. Biomaterials 121, 167-178 (2017).

17. Park, J.-H. et al. Magnetic Iron Oxide Nanoworms for Tumor Targeting and Imaging. Adv. Mater. 20, 1630-1635 (2008).

18. Harris, T. J. et al. Protease-triggered unveiling of bioactive nanoparticles. Small 4, 1307-1312 (2008).

19. Srinivasarao, M., Galliford, C. V. \& Low, P. S. Principles in the design of ligand-targeted cancer therapeutics and imaging agents. Nat. Rev. Drug Discov. 14, 203-219 (2015).

20. Ruoslahti, E., Bhatia, S. N. \& Sailor, M. J. Targeting of drugs and nanoparticles to tumors. J. Cell Biol. 188, 759-768 (2010).

21. Paradís-Bas, M., Tulla-Puche, J. \& Albericio, F. The road to the synthesis of 'difficult peptides'. Chem. Soc. Rev. 45, 631-654 (2016).

22. Tan, Y. N., Lee, J. Y. \& Wang, D. I. C. Uncovering the design rules for peptide synthesis of metal nanoparticles. J. Am. Chem. Soc. 132, 5677-5686 (2010).

23. Reshetnyak, Y. K., Andreev, O. A., Lehnert, U. \& Engelman, D. M. Translocation of molecules into cells by pH-dependent insertion of a transmembrane helix. Proc. Natl. Acad. Sci. U. S. A. 103, 6460-6465 (2006).

24. Lu, J. et al. Highly Sensitive Diagnosis of Small Hepatocellular Carcinoma Using pH-Responsive Iron Oxide Nanocluster Assemblies. J. Am. Chem. Soc. 140, 10071-10074 (2018).

25. Sugahara, K. N. et al. Coadministration of a tumor-penetrating peptide enhances the efficacy of 
cancer drugs. Science 328, 1031-1035 (2010).

26. Sugahara, K. N. et al. Tissue-penetrating delivery of compounds and nanoparticles into tumors. Cancer Cell 16, 510-520 (2009).

27. Araujo, A. C. V., Abreu, F., Silva, K. T., Bazylinski, D. A. \& Lins, U. Magnetotactic bacteria as potential sources of bioproducts. Mar. Drugs 13, 389-430 (2015).

28. Muxworthy Adrian R. \& Williams Wyn. Critical superparamagnetic/single-domain grain sizes in interacting magnetite particles: implications for magnetosome crystals. J. R. Soc. Interface 6, 1207-1212 (2009).

29. Krishnan, K. M. Biomedical Nanomagnetics: A Spin Through Possibilities in Imaging, Diagnostics, and Therapy. IEEE Trans. Magn. 46, 2523-2558 (2010).

30. Dunin-Borkowski, R. E. et al. Magnetic microstructure of magnetotactic bacteria by electron holography. Science 282, 1868-1870 (1998).

31. Kraupner, A. et al. Bacterial magnetosomes - nature's powerful contribution to MPI tracer research. Nanoscale 9, 5788-5793 (2017).

32. Lang, C., Schüler, D. \& Faivre, D. Synthesis of magnetite nanoparticles for bio- and nanotechnology: genetic engineering and biomimetics of bacterial magnetosomes. Macromol. Biosci. 7, 144-151 (2007).

33. Matsunaga, T., Suzuki, T., Tanaka, M. \& Arakaki, A. Molecular analysis of magnetotactic bacteria and development of functional bacterial magnetic particles for nano-biotechnology. Trends Biotechnol. 25, 182-188 (2007).

34. Borg, S., Hofmann, J., Pollithy, A., Lang, C. \& Schüler, D. New vectors for chromosomal integration enable high-level constitutive or inducible magnetosome expression of fusion proteins in Magnetospirillum gryphiswaldense. Appl. Environ. Microbiol. 80, 2609-2616 (2014).

35. Yao, L. et al. pHLIP peptide targets nanogold particles to tumors. Proc. Natl. Acad. Sci. U. S. A. 110, 465-470 (2013).

36. Kimbrough, C. W. et al. Targeting Acidity in Pancreatic Adenocarcinoma: Multispectral Optoacoustic Tomography Detects pH-Low Insertion Peptide Probes In Vivo. Clin. Cancer Res. 21, 4576-4585 (2015).

37. Teesalu, T., Sugahara, K. N. \& Ruoslahti, E. Tumor-penetrating peptides. Front. Oncol. 3, 216 (2013). 
38. O'handley, R. C. Modern magnetic materials: principles and applications. (Wiley, 2000).

39. Logozzi, M. et al. Microenvironmental pH and Exosome Levels Interplay in Human Cancer Cell Lines of Different Histotypes. Cancers 10, (2018).

40. Zhao, Y. et al. Tumor alphavbeta3 integrin is a therapeutic target for breast cancer bone metastases. Cancer Res. 67, 5821-5830 (2007).

41. Vellon, L., Menendez, J. A., Liu, H. \& Lupu, R. Up-regulation of alphavbeta3 integrin expression is a novel molecular response to chemotherapy-induced cell damage in a heregulin-dependent manner. Differentiation 75, 819-830 (2007).

42. Brown, M. A. et al. The use of mild trypsinization conditions in the detachment of endothelial cells to promote subsequent endothelialization on synthetic surfaces. Biomaterials $\mathbf{2 8}$, 3928-3935 (2007).

43. Knight, L. C., Romano, J. E., Cosenza, S. C., Iqbal, N. M. \& Marcinkiewicz, C. Differences in binding of 99mTc-disintegrins to integrin avß3 on tumor and vascular cells. Nucl. Med. Biol. 34, 371-381 (2007).

44. Taherian, A., Li, X., Liu, Y. \& Haas, T. A. Differences in integrin expression and signaling within human breast cancer cells. BMC Cancer 11, 293 (2011).

45. Wilhelm, S., Tavares, A. J., Dai, Q., Ohta, S. \& Audet, J. Analysis of nanoparticle delivery to tumours. Nat. Rev. (2016).

46. Harada, N. et al. Introduction of antisense CD44s cDNA down-regulates expression of overall CD44 isoforms and inhibits tumor growth and metastasis in highly metastatic colon carcinoma cells. International journal of cancer $\mathbf{9 1 , 6 7 - 7 5 ~ ( 2 0 0 1 ) . ~}$

47. Komeili, A., Vali, H., Beveridge, T. J. \& Newman, D. K. Magnetosome vesicles are present before magnetite formation, and MamA is required for their activation. Proc. Natl. Acad. Sci. U. S. A. 101, 3839-3844 (2004).

48. Okamura, Y. et al. Design and application of a new cryptic-plasmid-based shuttle vector for Magnetospirillum magneticum. Appl. Environ. Microbiol. 69, 4274-4277 (2003). 


\section{Figures}

\section{Magnetospirillum
magneticum Magnetosomes vivo tumor imaging}
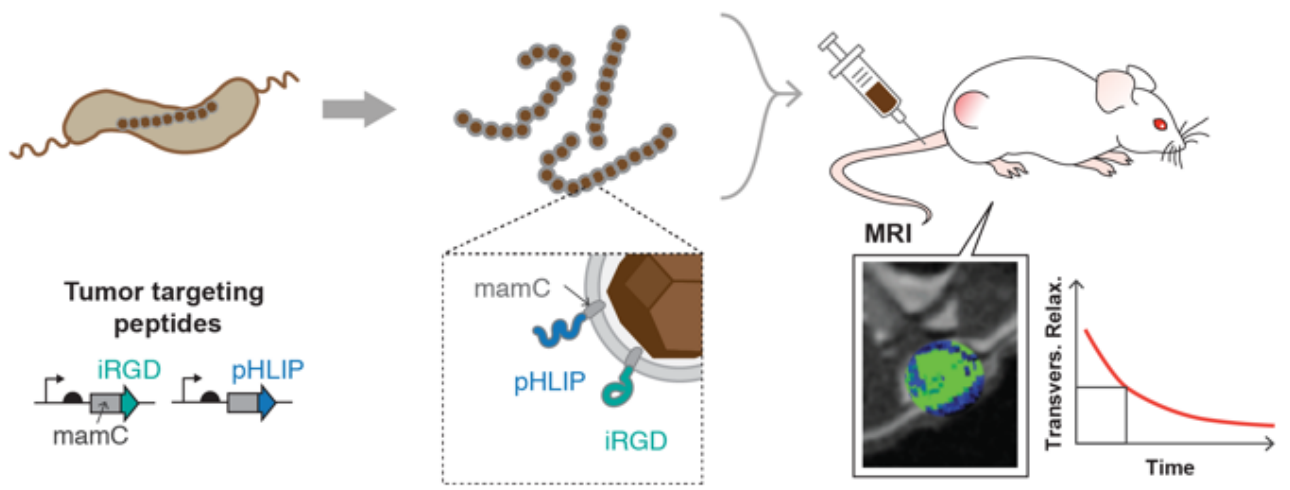

Graphical Abstract: Concept of genetically engineered magnetic nanoparticles with tumor targeting peptides as in vivo contrast agents 

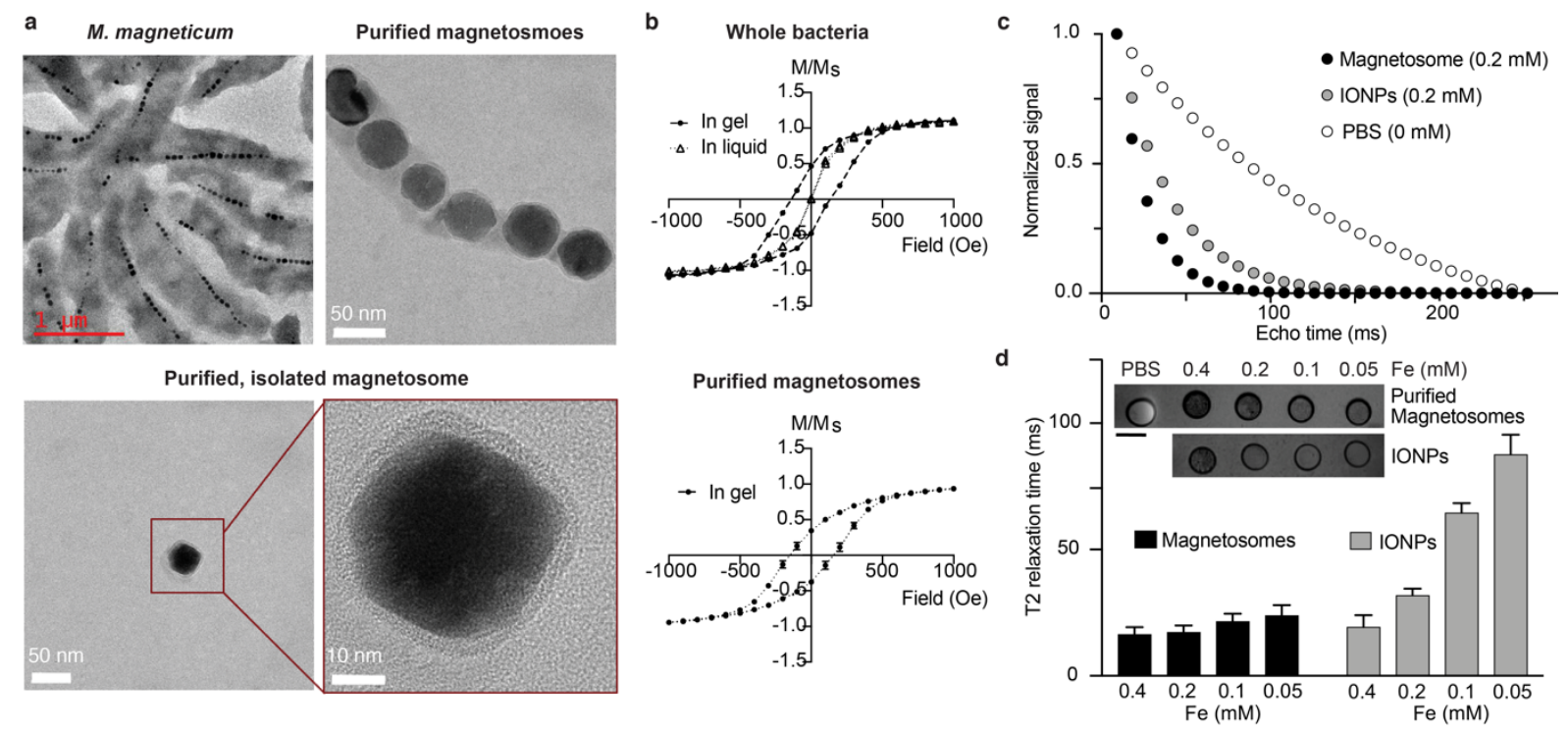

Figure 1. Magnetosomes are suitable T2 - weighted contrast agents. a) Transmission electron micrographs of $M$. magneticum before (left) and after (right) purification, showing an individual magnetosome. A zoomed-in TEM image of an isolated magnetosome core with lipid bilayer is shown in the bottom. b) The magnetic properties of intact AMB-1 cells (top) and purified magnetosomes (bottom) were characterized by vibrating sample magnetometry (VSM). Intact cells (top) were first measured in liquid and then immobilized in a gel to prevent physical rotation, allowing observation of their hysteresis ( $n=3$ samples of one culture). Purified magnetosomes showed similar behavior, indicating that the structural integrity of the magnetosomes was maintained. c) The determined magnetic properties suggested their use as T2-weighted contrast agents which was assessed in in T2relaxation time measurements and compared with samples of individually suspended IONPs with similar iron oxide nanoparticle core size $(25 \mathrm{~nm})$. Samples were immobilized in tubes containing agar and the shortening of T2 relaxation time (decay of signal amplitude to 1/e) was determined, yielding $17.3 \pm 2.6 \mathrm{~ms}$ for the magnetosomes, compared to $31.9 \pm 2.6 \mathrm{~ms}$ for $25 \mathrm{~nm}$ large iron oxide nanoparticles, for both samples at $0.2 \mathrm{mM} \mathrm{Fe}$ content. The mean is derived from the average T2 relaxation time per pixel in the measured region of interest. d) T2 relaxation time increases, and thus, the effect on T2 relaxation time reduction decreases, with decreasing iron concentration, as to be expected. While the commercial IONPs show a fairly linear increase with decreasing iron concentration, a muted decrease was observed for purified magnetosomes, which might be attributed to local clustering effects. The inset (top) shows the T2 intensity of cross-sectional scans of the tubes for the respective concentrations of the two samples. 
a

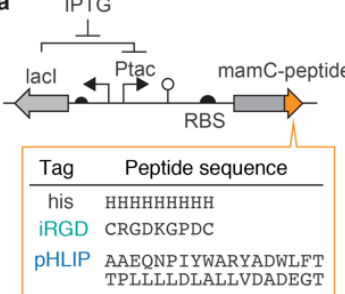

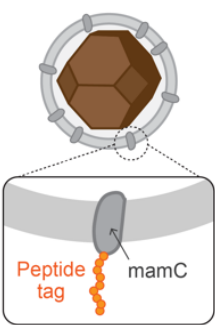

b

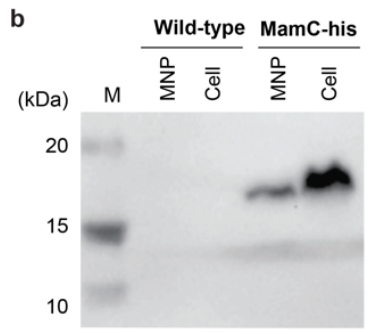

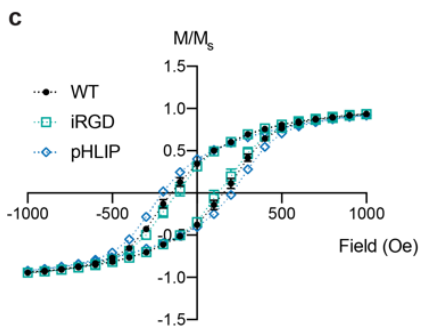

Figure 2. Genetic engineering of AMB-1. a) Peptide tag sequence (his-tag, iRGD, and pHLIP) was fused to the C-terminus of the mamC gene and placed under an IPTG-inducible $P_{\text {tac }}$ promoter. b) Western blot with anti-his antibody of magnetosome nanoparticle binding proteins ("MNP") or cell lysates ("Cell") extracted from wild-type or MamC-his expressing strain. c) The magnetic properties of peptide-fused magnetosome were assessed and compared to the previous measurements of the hysteresis of wild-type magnetosomes. The behavior is largely consistent, suggesting that the genetic alterations did not affect magnetosome synthesis by the bacterial cells. 

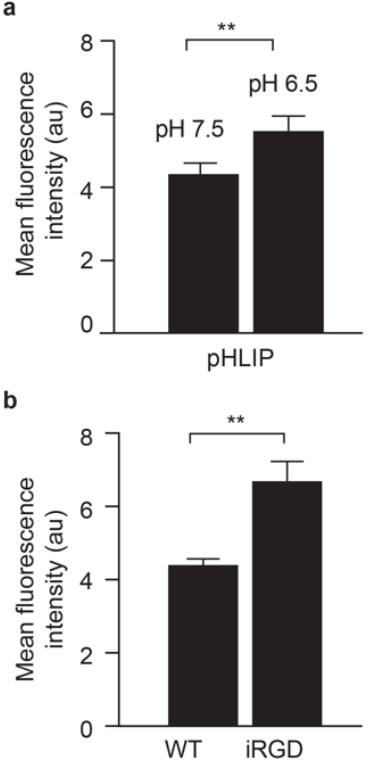

pHLIP pH 7.5

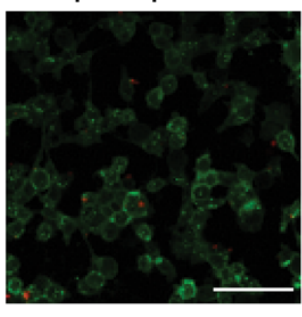

WT

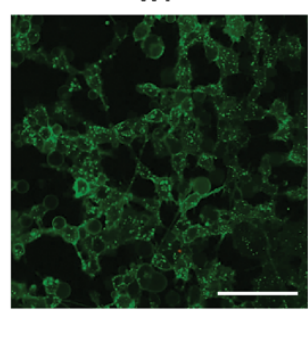

pHLIP pH 6.5

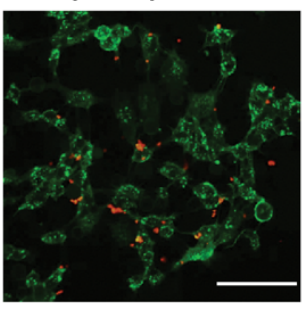

iRGD

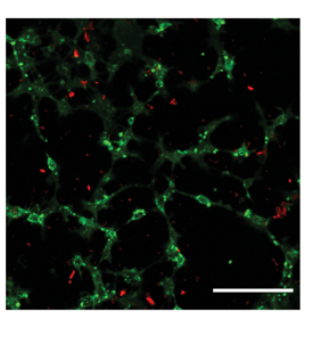

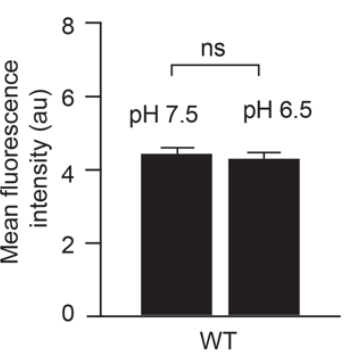

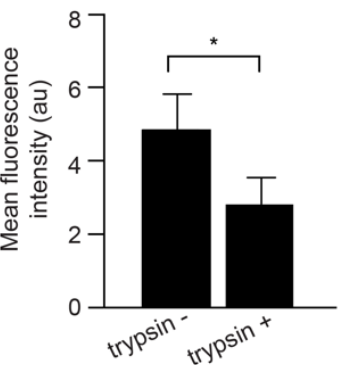

Figure 3. Magnetosomes functionalized with tumor-targeting peptides pHLIP and iRGD show increased binding affinity to cancer cells. a) pHLIP and wild-type magnetosomes were fluorescently labeled with a membrane dye (green) and incubated with MDA-MB-231 breast cancer cells at standard $(\mathrm{pH} 7.5)$ and low $\mathrm{pH}(\mathrm{pH}$ 6.5). Increased binding for $\mathrm{pHLIP}$ magnetosomes at low $\mathrm{pH}$ was measured by flow cytometry, compared to their binding at standard $\mathrm{pH}$ (left, $\mathrm{p}=0.0078$, unpaired, two sample $t$ test). This can be also seen in fluorescence images of cells (green) with magnetosomes (red) shown on the right (scale bars $=100 \mu \mathrm{m}$ ). No such $\mathrm{pH}$ dependency was observed for wild-type magnetosome (right, $p=0.6420$, unpaired, two sample t test) $b$ ) In another experiment, iRGD magnetosomes were incubated with the same cell line, known to express av integrins. A significantly increased selectivity to these cells was observed for iRGD magnetosomes compared to wild-type magnetosomes left, $p=0.0033$, unpaired, two sample t test), which is also reflected in the microscopy images on the right (scale bars $=100 \mu \mathrm{m}$ ). Binding efficiency significantly dropped when iRGD-functionalized magnetosomes were incubated with trypsinized cells, and thus bearing cleaved integrins, compared to untrypsinized cells with preserved integrin expression (right, $p=0.0411$, unpaired, two sample $t$ test). 

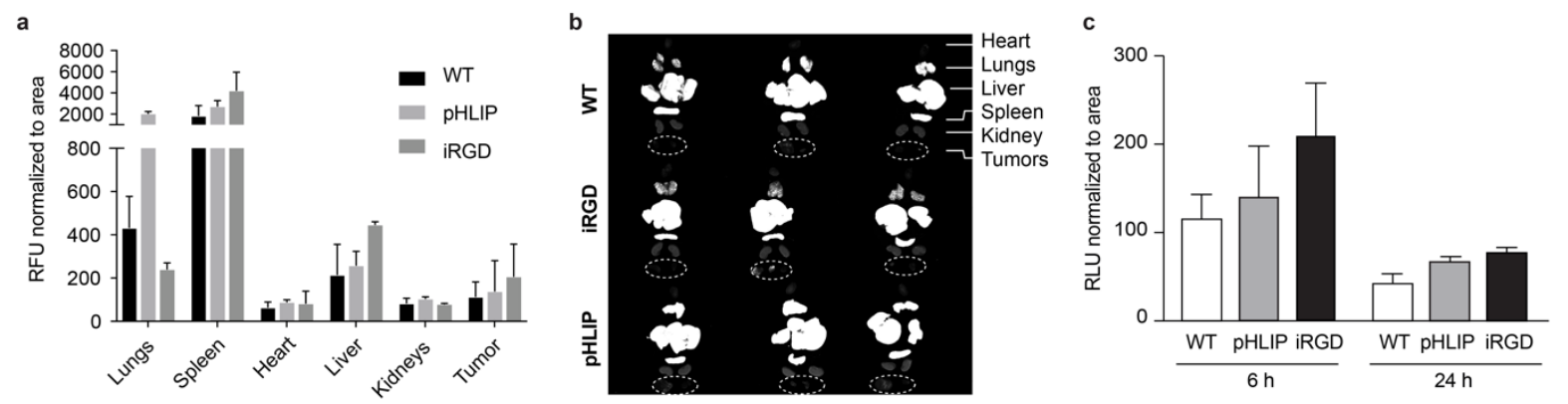

Figure 4. Peptide-fused magnetosomes show increased accumulation in tumors. a) Near infrared (NIR)-labeled (lipophilic dye Cy7.5) magnetosomes were intravenously injected into mice ( $n=3$ each group) bearing MDA-MB-435S flank tumors. Organs were harvested after $6 \mathrm{~h}$ and measured with a NIR scanner, with relative fluorescence units (RFU) normalized to the area of each organ. b) An intensity plot is shown for a scan at $800 \mathrm{~nm}$ emission wavelength on the harvested organs. Tumors are circled in white. c) Comparison of tumoral accumulation at $6 \mathrm{~h}$ and $24 \mathrm{~h}$ post injection. An increased accumulation of peptide displaying magnetosomes, although not statistically significant, was found in tumors compared to wildtype magnetosomes, with levels diminishing rapidly within $24 \mathrm{~h}$ ( $\mathrm{n}=3$ each group). 

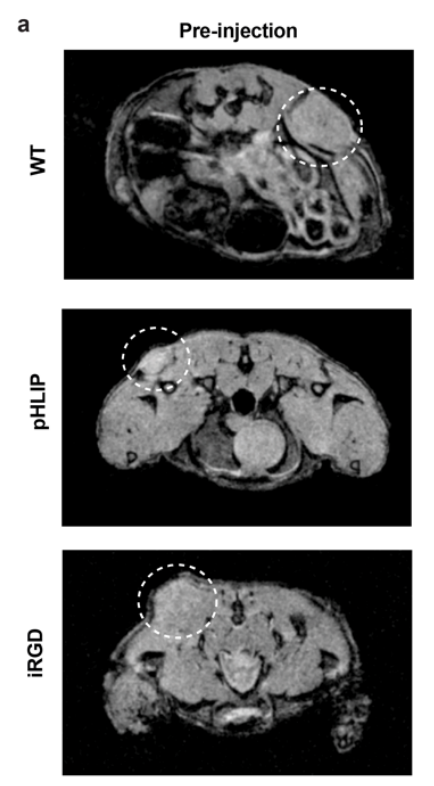

Post-injection
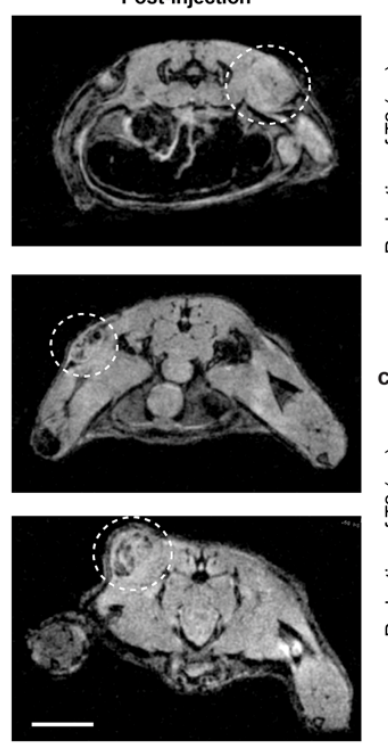

b
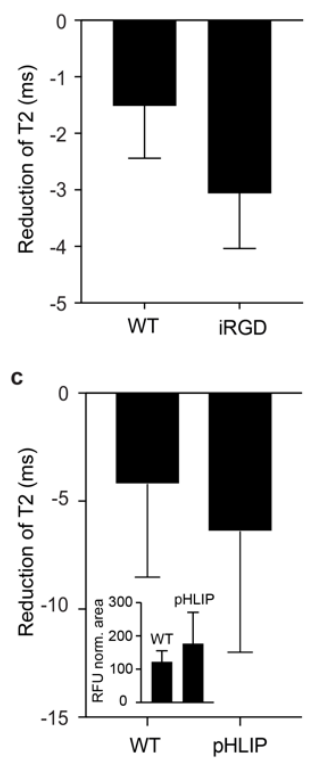

d
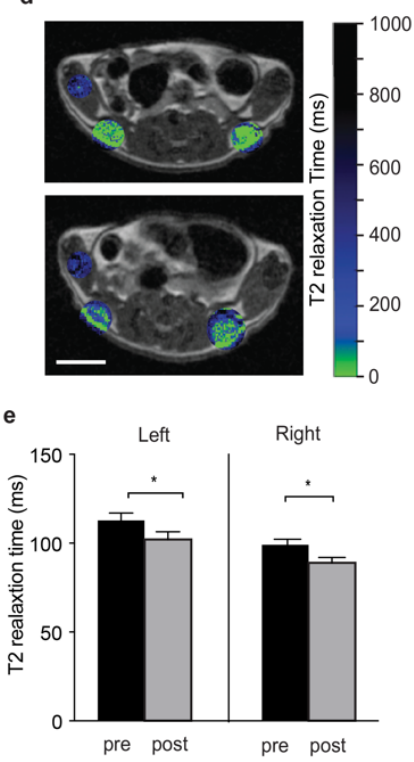

Figure 5. Peptide-fused magnetosomes show enhanced T2 weighted contrast in two different tumor models. a) Mice bearing MDA-MB-453S flank tumors were imaged in a 7T MRI scanner and T2-weighted relaxation time was determined before and after intravenous injection of magnetosomes. An enhanced darkening, attributable to a decrease in T2 relaxation time can be observed for peptidedisplaying magnetosomes relative to wild-type magnetosomes (scale bar $=10 \mathrm{~mm}$, same scale for all images, representative scans are shown for each group, $n=3$ each group). b) A 2.02-fold increase of the reduction of T2 relaxation was found across tumors in mice that were injected with iRGD magnetosomes compared to those exposed to wild-type magnetosomes $(p=0.277$, not significant according to unpaired, two-tailed student's t-test, $n=3$ animals, each, two flank tumors per animal). c) pHLIP functionalized magnetosomes were injected into mice bearing LS174t flank tumors and an increased tumor accumulation of pHLIP functionalized over non-functionalized magnetosomes $6 \mathrm{~h}$ post injection was observed (inset). Overall a T2 relaxation time reduction of $7.72 \%(6.40 \mathrm{~ms})$ was measured $(p=0.309$, not significant according to unpaired two tailed student's t-test, $n=3$ for the control and $n=4$ for the experimental group), demonstrating the potential of pHLIP displaying magnetosomes for T2 weighted in vivo tumor imaging. d) The magnitude of T2 relaxation time reduction was quantified and mapped in this example onto scans acquired by fast spin-echo multi-slice scan sequence (fsems) of a mouse with two flank tumors, injected with pHLIP magnetosomes (scale bar $=10 \mathrm{~mm}$, same scale for both images). The T2 relaxation time before and $5 \mathrm{~h}$ after injection is shown in $\mathbf{e}$ ). 